\title{
The Subthalamic Nucleus Contributes to Post-error Slowing
}

\author{
James F. Cavanagh ${ }^{1,2}$, Joseph L. Sanguinetti ${ }^{3}$, John J. B. Allen ${ }^{3}$, \\ Scott J. Sherman ${ }^{3}$, and Michael J. Frank ${ }^{2}$
}

\begin{abstract}
$\mathrm{pFC}$ is proposed to implement cognitive control via directed "top-down" influence over behavior. But how is this feat achieved? The virtue of such a descriptive model is contingent on a mechanistic understanding of how motor execution is altered in specific circumstances. In this report, we provide evidence that the well-known phenomenon of slowed RTs following mistakes (post-error slowing) is directly influenced by the degree of subthalamic nucleus (STN) activity. The STN is proposed to act as a brake on motor execution following conflict or errors, buying time so a more cautious response can be
\end{abstract}

\section{INTRODUCTION}

As our understanding of the nature of cognitive and executive control grows, increasingly fine-tuned descriptions of these processes have begun to emerge (Rushworth, Noonan, Boorman, Walton, \& Behrens, 2011; Buckley et al., 2009; Ridderinkhof, Ullsperger, Crone, \& Nieuwenhuis, 2004). It is widely believed that one means of implementing control involves directed "top-down" influence over prepotent or habitual actions, especially in difficult situations (Miller \& Cohen, 2001). Although this descriptive model is helpful, much work remains to be done to explain the distinct neural mechanisms by which such top-down control alters action selection. In this report, we provide evidence that the subthalamic nucleus (STN) contributes to the degree of RT slowing following an error.

Extensive evidence implicates the $\mathrm{pFC}$ in the realization of an error (Gehring, Liu, Orr, \& Carp, 2012; Botvinick, Braver, Barch, Carter, \& Cohen, 2001; Carter et al., 1998), yet the mechanistic details of how erroneous performance is resolved are less well specified. Post-error RT slowing is a well-known feature in cognitive accounts of performance monitoring (Botvinick et al., 2001; Gehring \& Fencsik, 2001; Rabbitt, 1966), whereupon the response following an error is slower and more accurate than the average response (Luce, 1986; Laming, 1979). The covary-

${ }^{1}$ University of New Mexico, ${ }^{2}$ Brown University, ${ }^{3}$ University of Arizona made on the next trial. STN local field potentials from nine Parkinson disease patients undergoing deep brain stimulation surgery were recorded while they performed a response conflict task. In a 2.5- to 5-Hz frequency range previously associated with conflict and error processing, the degree phase consistency preceding the response was associated with increasingly slower RTs specifically following errors. These findings provide compelling evidence that post-error slowing is in part mediated by a corticosubthalamic "hyperdirect" pathway for increased response caution. ing combination of slowed responses and increased accuracy is best represented by a single latent construct in formal models of performance: an increased decision threshold (Ratcliff \& McKoon, 2008; Luce, 1986). An increased decision threshold thus accounts for a shift in the speed-accuracy tradeoff toward increased response caution. One candidate neural system-the hyperdirect cortico-STN pathway-has been proposed to specifically act to increase decision threshold following signals of the need for control (Ratcliff \& Frank, 2012; Cavanagh et al., 2011; Frank, 2006).

The STN are small subcortical nuclei that lie between the brainstem and pallidum. Long considered a part of the corticostriatal indirect pathway, they have been implicated as a part of an inhibitory system that prevents motor gating, acting in antagonism to the facilitatory direct pathway (Mink, 1996; Alexander \& Crutcher, 1990). The existence of a distinct extrastriatal hyperdirect information processing stream has been supported by recent descriptions of the cortico-BG system including histological (Haynes \& Haber, 2013; Nambu, Tokuno, \& Takada, 2002; Nambu, Tokuno, \& Hamada, 2000), functional imaging (Mansfield, Karayanidis, Jamadar, Heathcote, \& Forstmann, 2011; Aron \& Poldrack, 2006), functional connectivity (Forstmann et al., 2012; Aron, Behrens, Smith, Frank, \& Poldrack, 2007), electrophysiological (Zaghloul et al., 2012; Cavanagh et al., 2011), and computational (Wiecki \& Frank, 2013; Ratcliff \& Frank, 2012; Frank, 2006) evidence. In the hyperdirect pathway, motor cortex and premotor cortex bypass the striatum and project 
directly to the STN, which in turn projects to the output nuclei of the BG (internal segment of the globus pallidus and substantia nigra pars reticulate $[\mathrm{SNr}]$ ) to act as a global brake on striatal output. This hyperdirect system is thus a compelling candidate "hold-your-horses" mechanism by which signals of conflict or error in the pFC could rapidly delay motor output via the STN, buying time for a more cautious decision (Frank, 2006).

Previous work has shown that high-frequency deep brain stimulation to the STN, which presumably disrupts STN processing of cortical inputs, induces more fast errors (Cavanagh et al., 2011; Wylie et al., 2010; Frank, Samanta, Moustafa, \& Sherman, 2007) and abolishes the normal relationship between enhanced mid-frontal EEG theta power and increased decision threshold (Cavanagh et al., 2011). Similarly, STN spiking increases with decision conflict and is predictive of higher accuracy and slowed RTs (Zaghloul et al., 2012), in line with an increased decision threshold. As may be expected, post-error slowing has been formally and specifically associated with a strategic increase in decision threshold (Dutilh et al., 2012), providing a strong rationale for a likely role of the STN in this feature of adaptive control. Although many previous investigations of STN activity have highlighted the roles of beta ( $\sim 12$ to $25 \mathrm{~Hz})$ and gamma $(\sim 55$ to $75 \mathrm{~Hz})$ bands during action readiness and selection (Jenkinson \& Brown, 2011; Marceglia, Fumagalli, \& Priori, 2011; Androulidakis et al., 2007; Weinberger et al., 2006; Kühn et al., 2004), conflict and errors have been specifically associated with lower-frequency responses sharing a 3-5 Hz range (Alegre et al., 2013; Zavala et al., 2013; Brittain et al., 2012; Cavanagh et al., 2011), motivating a candidate frequency range that may be sensitive to control-related adjustment. In this investigation, we provide evidence from Parkinsonian patients undergoing surgery for deep brain stimulation that STN activities in this $2.5-5 \mathrm{~Hz}$ frequency range specifically contribute to the degree of post-error slowing.

\section{METHODS}

\section{Participants}

Eleven volunteers participated in the task during the implantation of the first deep brain stimulation electrode: two were rejected for poor data quality, leaving nine participants in total. The average age was 74 years $(S D=$ 7.9, range $=59-86$ ); seven were men. All participants completed informed consent approved by the Tucson Medical Center Institutional Review Board. All participants were off L-DOPA medication for at least $12 \mathrm{hr}$ before surgery, although patients remained on other non-Parkinsonian medications as well as local anesthetic.

\section{Task}

A modified Simon task (Cavanagh, Zambrano-Vazquez, \& Allen, 2012; Simon \& Rudell, 1967) with preparatory cues was used to assess response competition processes (Figure 1A). Participants learned the task rules and practiced at least 38 trials in their hospital room $2 \mathrm{hr}$ before the surgery. Each trial began with an informative cue (green "EASY," red "HARD," or purple "XXXX"), indicating that the trial would require a congruent or incongruent response or, in the case of purple Xs, that the response was equiprobably congruent or incongruent. These cues are hereafter referred to as informative cues, given that they provide information (EASY, HARD) or no information (XXXX) about the upcoming trial. Previous work has shown that noninformative cues are associated with

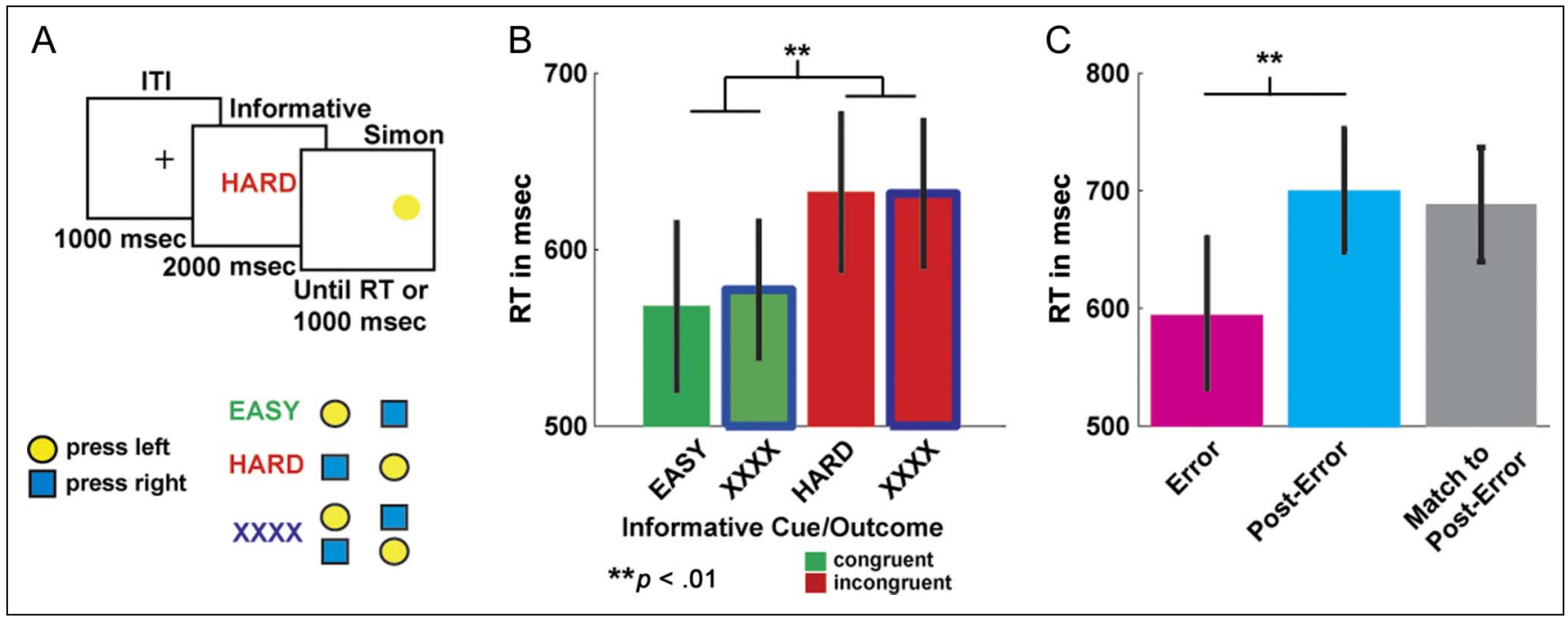

Figure 1. Task and performance. (A) Participants performed a Simon task with informative versus noninformative cues. (B) Although there was no behavioral evidence that participants used the informative cues, there was a strong effect of trial congruency on RT (error bars are SEM). (C) Aggregated RTs showing significant post-error slowing (error bars are 95\% confidence intervals), with the comparison condition of correct RTs matched to the post-error trial to investigate post-error specific slowing. 
increased mid-frontal signals of conflict (Cavanagh et al., 2012); here we aimed to investigate if these cues were also associated with increased STN activity. Informative cues were presented for $2000 \mathrm{msec}$, after which the imperative Simon cue was presented to the left or right side of the screen (yellow circle for left response, blue square for right response) for $1000 \mathrm{msec}$ or until the response. These imperative cues were thus either spatially congruent (screen side $=$ response hand) or incongruent (screen side $\neq$ response hand) as in a standard Simon task. Erroneous responses were followed by a delay of $1000 \mathrm{msec}$ followed by "Incorrect" feedback presented for $1000 \mathrm{msec}$, and nonresponses were followed by "Faster!" feedback immediately presented for $1000 \mathrm{msec}$. All trials had an intertrial interval of $1000 \mathrm{msec}$ before the onset of the next informative cue. There were 40 trials of each separate informative-imperative pair (EASY-congruent, HARDincongruent, XXXX-congruent, XXXX-incongruent). All conditions were randomly presented and were counterbalanced between equal numbers of yellow versus blue stimuli, right versus left responses, and so forth.

\section{Intracranial EEG Recording and Processing}

Intracranial EEG (iEEG) recording and data analysis were similar to our previous study (Cavanagh et al., 2011). Field activity was recorded from a Medtronic 3387 (Minneapolis, MN) stimulating electrode using a Synamps ${ }^{2}$ system (bandpass filter $0.05-500 \mathrm{~Hz}, 2000 \mathrm{~Hz}$ sampling rate) referenced to the right mastoid site and grounded on the collarbone. All STN recordings were taken from the left STN because this was always the first electrode implanted. Electrode placement was determined by the surgical staff based on preoperative stereotaxic planning, the firing pattern from the microelectrode recordings, and immediate clinical effectiveness of stimulation. The surgical team sought to place the quadripolar electrode so that the distal (ventral) contact corresponded to the ventral boundary of the STN as determined by microelctrode recordings and immediate motor improvement during stimulation. The Medtronic electrode included four contacts, which were bipolar referenced (three pairs ranging from ventral to dorsal), resulting in three separate recordings of STN area activity. These recordings are referred to by their proximal location to each other: ventral, middle, and dorsalalthough their exact location in regard to subnuclei of the STN is unknown.

Data were epoched around the informative cues $(-1500$ to $5500 \mathrm{msec})$ and baseline-corrected to the precue average ( -500 to $-300 \mathrm{msec})$. Epochs with bad signal recordings were manually rejected (2\% of trials), and data were downsampled to $500 \mathrm{~Hz}$. Time-frequency calculations were computed using custom-written Matlab (The MathWorks, Natick, MA) routines (Cavanagh, Cohen, \& Allen, 2009). Time-frequency measures were computed by multiplying the fast Fourier transformed (FFT) power spectrum of single-trial EEG data with the FFT power spectrum of a set of complex Morlet wavelets and taking the inverse FFT. The wavelet family is defined as a set of Gaussian-windowed complex sine waves: $e^{-i 2 \pi f f} e^{\left.-t^{2} / 2^{*} \sigma 2\right)}$, where $t$ is time, $f$ is frequency (which increased from 1 to $50 \mathrm{~Hz}$ in 50 logarithmically spaced steps), and $\sigma$ defines the width (or "number of cycles") of each frequency band, set according to $4 /(2 \pi f)$. The end result of this process is identical to time domain signal convolution. No effects were found above $50 \mathrm{~Hz}$, so the time-frequency plots reported here focus on the $1-50 \mathrm{~Hz}$ range.

Power was defined as $Z[t]$ (power time series: $p(t)=$ $\left.\operatorname{real}[z(t)]^{2}+\operatorname{imag}[z(t)]^{2}\right)$ and was normalized for display by conversion to a decibel scale based on the average prestim baseline defined above $(10 \times \log 10[\operatorname{power}(t) /$ power(baseline)]), allowing a direct comparison of effects across frequency bands. The phase angle was defined as $\varphi_{t}=\arctan (\operatorname{imag}[z(t)] / \mathrm{real}[z(t)])$. Intertrial phase coherence (also termed the phase locking value) was used to measure the consistency of phase values for a given frequency band at each point in time (Lachaux, Rodriguez, Martinerie, \& Varela, 1999). Intertrial phase coherence values vary from 0 to 1 , where 0 indicates random phases at that time-frequency point across trials and 1 indicates identical phase values at that time-frequency point across trials. Given that the cue-locked investigations of noninformative versus informative cues and the responselocked power analyses were nonsignificant, we focus on the methods of the response-locked phase modulation described in Figure 3.

\section{Statistical Analysis}

First, correct RTs and errors were tested in separate 2 (Information [EASY and HARD] vs. no information $[\mathrm{XXXX}]) \times 2$ (Conflict: congruent vs. incongruent) general linear models (GLMs) to investigate the effect of the conditions on aggregate performance. There were too few errors per participant $($ median $=6$ [3.75\%], range $=2-18$ [1.25$11.25 \%]$ ) for standard fixed-effect analyses across participants. Given the rarity of response errors and the absence of a human STN study of response errors, we utilized an alternative statistical procedure by aggregating all trials into a single data set. Before aggregation, each participant's RTs were $z$-scored, and iEEG data were timefrequency transformed as described above and locked to the response ( -500 to $500 \mathrm{msec}$ ), whereupon each cell in the time-frequency power matrix was $z$ scoretransformed across trials. Although this $z$-scoring procedure was utilized to control for between-participant differences in base rate RT and amplitude, findings were highly similar using the original microvolt-scaled EEG instead of $z$-scored EEG.

Error trials $(n=77)$ and valid post-error trials $(n=72$; there were fewer because of four nonresponses and one error at the very end of the experiment) were identified. Then, a matched data set of correct trials was selected based on the nearest $\mathrm{RT} z$ score match to the post-error 
trial (to contrast with slowing specifically following errors; see Figure 1C). Matched correct trials were limited to responses that were not immediately preceding errors or following post-error trials. These sets of normalized RTs and normalized time-frequency transformed iEEG activities were then aggregated across participants, yielding $n=72$ post-error and matched correct trials (trials per participant: $[2,4,4,4,5,10,12,14,17])$. There were similar numbers of congruent and incongruent post-error trials (36 each) and matched trials (35 vs. 37). There were no meaningful differences in the handedness of trials for errors (56\% right), post-error trials (48\% right), and matched correct trials (50\% right). Although these conditions were very well matched on RT, handedness, and congruency, the differentiation due to previous trial type was also influenced by an increased delay following errors due to the presentation of "Incorrect" feedback (although we do not think that this delay per se contributed to the effects reported here).

Statistical differences between error and post-error RT conditions were assessed based on confidence intervals estimated by bootstrapping the mean of each distribution 1000 times; statistical significance was determined by transforming confidence intervals to $z$ score (Altman \& Bland, 2011). Spearman's $\rho$ correlations were performed at each time-frequency point to test the relationship between normalized spectral power and the normalized RT on the post-error and matched correct trials. Conditionspecific differences between these correlations were tested using $\rho$-to- $z$ transforms. Although correlations can be used to investigate power-RT relationships, we were also interested in the relationship between RT and phase consistency. The influence of phase consistency cannot be assessed with linear correlations, as these data are circularly distributed. On the basis of a similar methods as phase-amplitude coupling (Canolty et al., 2006), the single trial influence of RT on phase consistency can be investigated by taking each RT-phase pair as a vector in complex space with the phase as the angle and the RT as the modulus (absolute value), as detailed in Cohen and Cavanagh (2011). The magnitude of the averaged complex data thus reflects the modulation of RT by phase angle, such that any relationship would indicate that phase consistency changes as a function of RT. For these analyses, $z$-scored RTs were offset by the minimum $z$-scored RT value plus a small constant, as negative values cannot be used.

To account for the potential influence of a noneven distribution of phase values across trials (i.e., because of response-locked phase reset), the phase modulation between 1000 bootstrapped (selection with replacement) trials and 1000 permuted (random shuffling of trial label) RTs were computed at each time-frequency point within each condition. These distributions were used to normalize the empirical magnitude of phase-RT modulation (by computing the difference between the empirical and bootstrapped means normalized by the standard devia- tion across these bootstrapped distributions). This procedure created a modulation index (MI), identical to a $z$-scored difference from the permutation-tested null hypothesis (Cohen \& Cavanagh, 2011; Canolty et al., 2006). Condition-specific differences were computed as the difference in MI.

To control for multiple comparisons, power correlations were rerun 1000 times with permuted RTs. Because each phase-modulated RT analyses required 1000 bootstrapped trials to compute the empirical MI, these bootstrapped trials were simply permuted by randomly selecting a new condition label (post-error or matched correct) 1000 times to create 1000 new "null" distributions. A random trial was selected to stand in for the empirical magnitude for each of the 1000 permuted distributions, facilitating the calculation of 1000 permuted MIs. For both power and phase modulation permutations, the maximum significant cluster size in the a priori 2.5-5 Hz range was saved for each of the 1000 permutations, creating a distribution of significant clusters that could be expected to occur because of chance. The 95th percentile of this distribution was used as the empirical threshold to provide a two-tailed 5\% level of control for multiple comparisons within a priori defined timefrequency space.

\section{RESULTS \\ Performance}

The repeated-measures GLM for RT revealed a main effect for Congruency, $F(1,8)=23.96, p<.01$, with no main or interactive effects for Information. Figure $1 \mathrm{~B}$ shows that incongruent trials were specifically associated with slower responses. The repeated-measures GLM for error rates also only revealed a main effect for Congruency, $F(1,8)=6.62, p<.05$, with more errors on incongruent trials than congruent trials. Participant age significantly correlated with error rate, $\rho(9)=.68, p<.05$, but not RT, $\rho(9)=-.07$, ns. As shown in Figure 1C, there was significant post-error slowing when measured in milliseconds $(z=2.52, p=.01)$ or as aggregate $z$ scores $(z=3.54, p<.01)$.

\section{iEEG}

Figure 2 shows the grand-averaged power and phase consistency for responses at the individual bipolar leads in the STN area. ERPs are shown on the power plots (black lines in overlay), detailing a large response-locked voltage negativity in the ventral STN area. Responses were characterized by enhanced power before (dorsal, middle) and during (ventral) the responses, with enhanced lowfrequency phase consistency following the response in all channels. There were no significant differences in timefrequency power between post-error and RT matched conditions. 


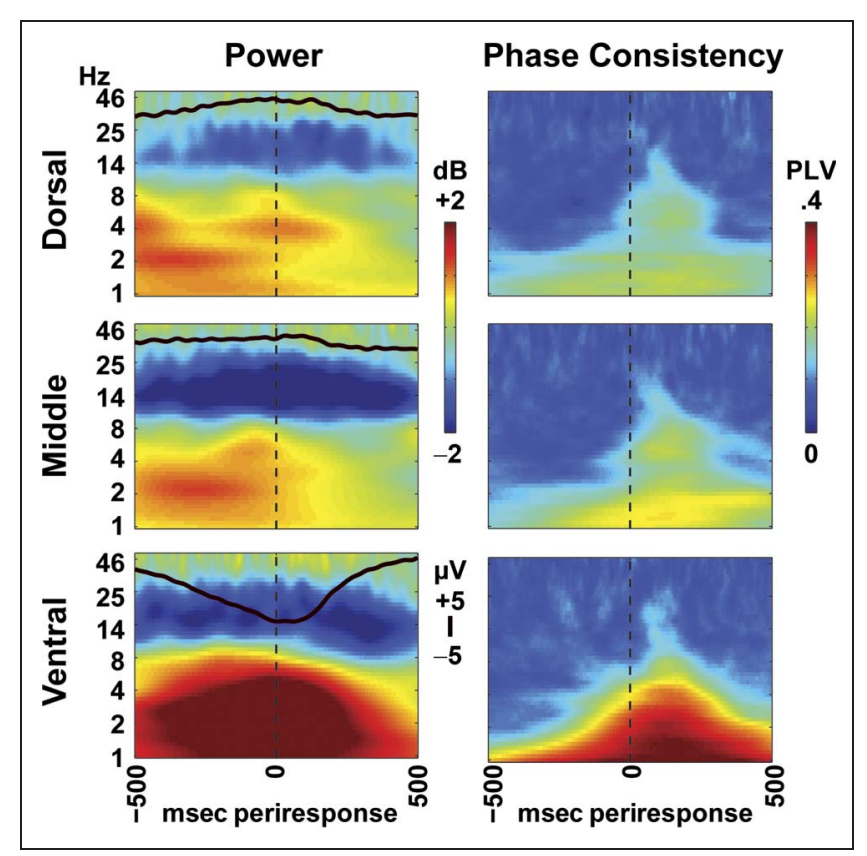

Figure 2. Response-locked power and phase consistency at the three bipolar leads in the STN area. Time-frequency plots show beta band power suppression and low-frequency enhancement before (dorsal, middle) and surrounding (ventral) the response (at $0 \mathrm{msec}$ ). ERPs are overlapped in black, showing a very large response-locked negative voltage deflection in the ventral lead. Phase consistency was enhanced surrounding (ventral) and following (dorsal, middle) the response. PLV $=$ phase locking value.

\section{iEEG Relationships with RT}

The correlation between STN area power and RT was examined within the post-error and matched correct conditions. There were no significant differences between post-error and matched correct correlations that survived multiple comparisons correction. Using the RT-weighted phase consistency approach described above, Figure 3 demonstrates that middle STN areas exhibited enhanced $2.5-5 \mathrm{~Hz}$ phase consistency before the response as a function of longer RTs. These patterns held up when contrasted to the matched RT condition. These findings demonstrate that the STN appears to be related to posterror specific RT slowing because of an enhanced preresponse low-frequency phase consistency during longer and presumably more deliberative gating of responses during a speed-accuracy tradeoff.

\section{DISCUSSION}

The findings reported here implicate the STN in the degree of deliberative speed-accuracy tradeoff following response errors, providing further evidence for the role of the STN during the strategic increase of decision threshold. These current findings extend previous manipulative (Cavanagh et al., 2011; Wylie et al., 2010; Frank et al., 2007) and correlative (Alegre et al., 2013; Zavala et al., 2013; Cavanagh et al., 2011) findings by demonstrating that $2.5-5 \mathrm{~Hz}$ STN activity is involved in the degree of slowing following errors-a well-known speed-accuracy tradeoff that has been formally associated with a strategic increase in decision threshold (Dutilh et al., 2012).

\section{Low-frequency STN Activities and Adaptive Control}

In its role in the hyperdirect pathway, the STN is proposed to act as a brake on striatal output particularly following cortical signals of conflict or error. It is known that cingulate and premotor areas preferentially respond
Figure 3. Time-frequency plots of RT-modulated phase consistency in the middle STN lead to responses (at $0 \mathrm{msec}$ ) on post-error trials (left column), matched correct trials (middle column), and the difference between these measures (right column). Values are presented as the MI, where higher values indicate greater phase consistency associated with longer responses. On the post-error trial, longer responses were associated with more 2.5-5 Hz phase consistency. When compared with RT-matched trials, these band-specific directional effects were maintained. Below, linear plots of MI for the $2.5-5 \mathrm{~Hz}$ frequency range are shown for each condition; horizontal dashed lines indicate statistical significance.

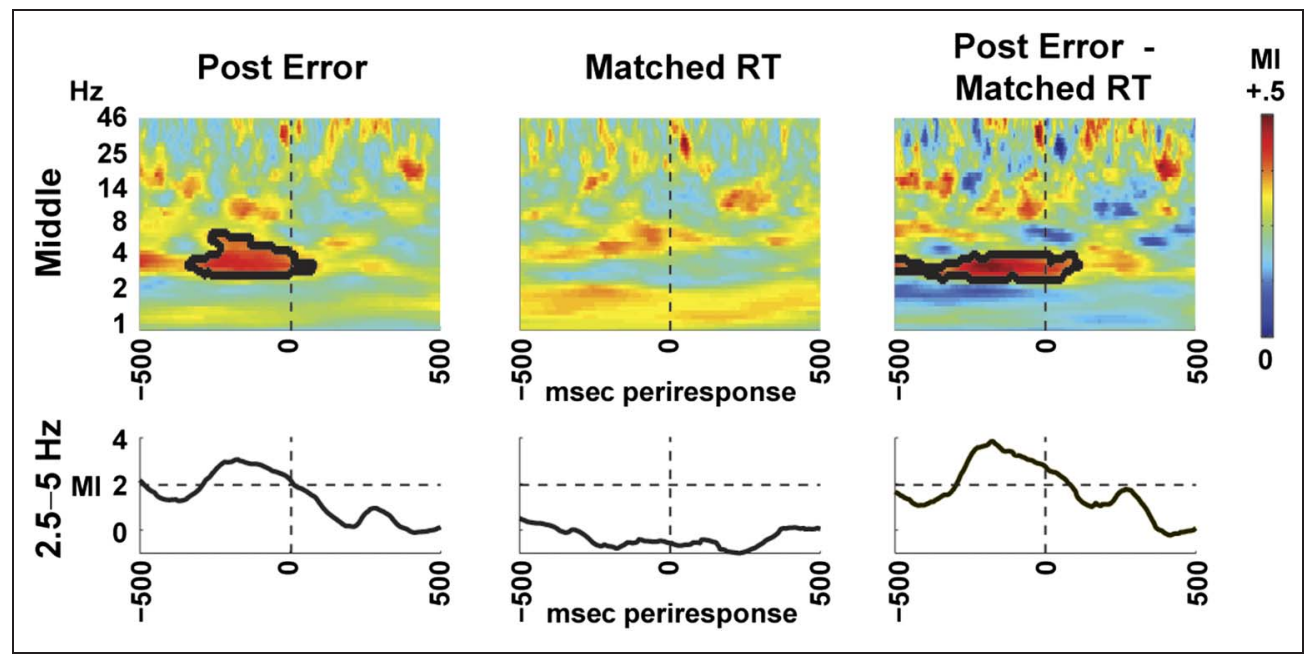


to errors and that theta band ( $\sim 4$ to $8 \mathrm{~Hz}$ ) activities from these regions are particularly associated with the realization and resolution of error and conflict (Cavanagh et al., 2009; Debener et al., 2005). Given previous findings of $2.5-4.5 \mathrm{~Hz}$ STN activities during conflict (Cavanagh et al., 2011) and $2.5-5 \mathrm{~Hz}$ activities during error (Alegre et al., 2013), it was expected that a similar range of activities would be associated with the mechanism of conflicterror resolution via slowed RT. Evidence from single neuron spiking in both monkey and human STN however has revealed strong evidence for preresponse activity related to conflict and associated with delayed choices and improved accuracy (Zaghloul et al., 2012; Isoda \& Hikosaka, 2008), similar to computational models (Wiecki \& Frank, 2013; Ratcliff \& Frank, 2012). Indeed, a very recent report has identified how periresponse 3-8 $\mathrm{Hz}$ power and preresponse $4-8 \mathrm{~Hz}$ phase consistency are modulated in the STN by conflict (Zavala et al., 2013). However, unlike the current report, preresponse phase consistency was enhanced for conflict trials with faster RTs. Although there are many differences between these investigations, a common possibility is that preresponse phase consistency $\sim 4 \mathrm{~Hz}$ is enhanced when STN is particularly active: rapidly for successful inhibition of inappropriate response tendencies yet slowly for deliberative control over the speed-accuracy tradeoff.

\section{Limitations to the Current Study}

Although we refer to the STN leads by their proximal location to each other (ventral, middle, and dorsal), their exact location with regard to subnuclei of the STN is unknown. However, this terminology still reflects the most bias-free way of describing the leads in relation to each other. Electrode placement was determined by microwire recordings and postimplantation motor improvement to stimulation. Experimentation immediately followed the determination of successful STN localization, yet all these recordings were from the left STN. Although there were no handedness differences between conditions reported here, future investigations may reveal hemispheric differences in the contribution of the STN to cognitive behavioral control.

Curiously, as observed in Figure 2, activities within the ventral lead were characterized by very strong power and phase dynamics surrounding the response, particularly observed as a strong negative deflection in the ERP that peaked at the time of the response. This pattern suggests not only that the ventral lead activities reflect an aspect of action execution but also that this lead may have even been capturing activities from the immediately ventral neighbor of the STN, the SNr, which acts as an output nucleus involved in gating/executing BG output. Given that no postoperative MRIs were obtained, it remains unknown whether this lead truly reflected STN activities. Future studies may be able to assist in interpretation of the localization of the results observed here in the middle lead if strong response-locked ERPs are observed immediately ventrally from known STN or SNr nuclei.

\section{Analytic Methods Motivated by the Experimental Environment}

Although we observed clear post-error-related activities within the STN leads, there were no findings for the uninformative versus informative cues, which have previously been associated with increased mid-frontal theta power and RT slowing (Cavanagh et al., 2012). Given that there was no effect of RT slowing for these cues here (Figure 1B), these patients may not have used informative cues to adapt their performance, obviating any potential effects in the iEEG. In the absence of those behavioral effects amenable to standard statistical procedures, this experiment utilized a novel method for aggregating small amounts of error-related data across participants to assess relationships in events that would otherwise be too rare to be informative. The existence of strong post-error slowing across participants (Figure 1C) provided a strong rationale that this facet of adaptive control remained intact during the task. The procedures for standardizing EEG power and RTs within participants facilitated data aggregation while allowing for appropriate contrasts to examine slowing that was specific to post-error adaptations (by contrasting correct RTs matched to the post-error RT). However, the distribution of errors was not uniform across participants and was positively correlated with the age of participants. It is unknown how these dynamics may affect the findings reported here, but these issues would similarly affect a standard fixed effects analysis of the results. Importantly, the replication of the precise temporofrequency range of previous conflict and error effects (Alegre et al., 2013; Zavala et al., 2013; Brittain et al., 2012; Cavanagh et al., 2011) lend strong confidence to the validity of these findings from this rare and challenging experimental scenario.

\section{Conclusion}

In a $2.5-5 \mathrm{~Hz}$ frequency range previously associated with conflict and error processing, STN power following the response and phase consistency preceding the response were associated with increasingly slower RTs specifically following errors. These findings provide compelling evidence that post-error slowing is in part mediated by a corticosubthalamic hyperdirect pathway for increased response caution.

\section{Acknowledgments}

The authors express their gratitude to T. Norton and his surgical staff for their support during the intraoperative recording sessions. This project was supported by NIH grant RO1 MH080066-01 and NSF grant 1125788 
Reprint requests should be sent to Dr. James F. Cavanagh, Department of Psychology, University of New Mexico, 2001 Redondo Dr NE, Albuquerque, NM 87131, or via e-mail: jcavanagh@unm.edu.

\section{REFERENCES}

Alegre, M., Lopez-Azcarate, J., Obeso, I., Wilkinson, L., Rodriguez-Oroz, M. C., Valencia, M., et al. (2013). The subthalamic nucleus is involved in successful inhibition in the stop-signal task: A local field potential study in Parkinson's disease. Experimental Neurology, 239, 1-12.

Alexander, G. E., \& Crutcher, M. D. (1990). Functional architecture of basal ganglia circuits: Neural substrates of parallel processing. Trends in Neurosciences, 13, 266-271.

Altman, D. G., \& Bland, J. M. (2011). How to obtain the $\mathrm{P}$ value from a confidence interval. BMJ, 343, d2304.

Androulidakis, A. G., Kühn, A. A., Chen, C. C., Blomstedt, P., Kempf, F., Kupsch, A., et al. (2007). Dopaminergic therapy promotes lateralized motor activity in the subthalamic area in Parkinson's disease. Brain, 130, 457-468.

Aron, A. R., Behrens, T. E., Smith, S., Frank, M. J., \& Poldrack, R. A. (2007). Triangulating a cognitive control network using diffusion-weighted magnetic resonance imaging (MRI) and functional MRI. Journal of Neuroscience, 27, 3743-3752.

Aron, A. R., \& Poldrack, R. A. (2006). Cortical and subcortical contributions to Stop signal response inhibition: Role of the subthalamic nucleus. Journal of Neuroscience, 26, 2424-2433.

Botvinick, M. M., Braver, T. S., Barch, D. M., Carter, C. S., \& Cohen, J. D. (2001). Conflict monitoring and cognitive control. Psychological Review, 108, 624-652.

Brittain, J.-S., Watkins, K. E., Joundi, R. A., Ray, N. J., Holland, P., Green, A. L., et al. (2012). A role for the subthalamic nucleus in response inhibition during conflict. Journal of Neuroscience, 32, 13396-13401.

Buckley, M. J., Mansouri, F. A., Hoda, H., Mahboubi, M., Browning, P. G. F., Kwok, S. C., et al. (2009). Dissociable components of rule-guided behavior depend on distinct medial and prefrontal regions. Science, 325, 52-58.

Canolty, R. T., Edwards, E., Dalal, S. S., Soltani, M., Nagarajan, S. S., Kirsch, H. E., et al. (2006). High gamma power is phase-locked to theta oscillations in human neocortex. Science, 313, 1626-1628.

Carter, C. S., Braver, T. S., Barch, D. M., Botvinick, M. M., Noll, D., \& Cohen, J. D. (1998). Anterior cingulate cortex, error detection, and the online monitoring of performance. Science, 280, 747-749

Cavanagh, J. F., Cohen, M. X., \& Allen, J. J. B. (2009). Prelude to and resolution of an error: EEG phase synchrony reveals cognitive control dynamics during action monitoring. Journal of Neuroscience, 29, 98-105.

Cavanagh, J. F., Wiecki, T. V., Cohen, M. X., Figueroa, C. M., Samanta, J., Sherman, S. J., et al. (2011). Subthalamic nucleus stimulation reverses mediofrontal influence over decision threshold. Nature Neuroscience, 14, 1462-1467.

Cavanagh, J. F., Zambrano-Vazquez, L., \& Allen, J. J. B. (2012). Theta lingua franca: A common mid-frontal substrate for action monitoring processes. Psychophysiology, 49, 220-238.

Cohen, M. X., \& Cavanagh, J. F. (2011). Single-trial regression elucidates the role of prefrontal theta oscillations in response conflict. Frontiers in Psychology, 2, 30

Debener, S., Ullsperger, M., Siegel, M., Fiehler, K., von Cramon, D. Y., \& Engel, A. K. (2005). Trial-by-trial coupling of concurrent electroencephalogram and functional magnetic resonance imaging identifies the dynamics of performance monitoring. Journal of Neuroscience, 25, 11730-11737.
Dutilh, G., Vandekerckhove, J., Forstmann, B. U., Keuleers, E., Brysbaert, M., \& Wagenmakers, E.-J. (2012). Testing theories of post-error slowing. Attention, Perception, E Psychophysics, 74, 454-465.

Forstmann, B. U., Keuken, M. C., Jahfari, S., Bazin, P.-L., Neumann, J., Schäfer, A., et al. (2012). Cortico-subthalamic white matter tract strength predicts interindividual efficacy in stopping a motor response. Neuroimage, 60, 370-375.

Frank, M. J. (2006). Hold your horses: A dynamic computational role for the subthalamic nucleus in decision making. Neural Networks, 19, 1120-1136.

Frank, M. J., Samanta, J., Moustafa, A. A., \& Sherman, S. J. (2007). Hold your horses: Impulsivity, deep brain stimulation, and medication in parkinsonism. Science, 318, 1309-1312

Gehring, W. J., \& Fencsik, D. E. (2001). Functions of the medial frontal cortex in the processing of conflict and errors. Journal of Neuroscience, 21, 9430-9437.

Gehring, W. J., Liu, Y., Orr, J. M., \& Carp, J. (2012). The error-related negativity (ERN/Ne). In S. J. Luck \& E. Kappenman (Eds.), Oxford handbook of event-related potential components (pp. 231-291). New York: Oxford University Press.

Haynes, W. I. A., \& Haber, S. N. (2013). The organization of prefrontal-subthalamic inputs in primates provides an anatomical substrate for both functional specificity and integration: Implications for basal ganglia models and deep brain stimulation. Journal of Neuroscience, 33, 4804-4814.

Isoda, M., \& Hikosaka, O. (2008). Role for subthalamic nucleus neurons in switching from automatic to controlled eye movement. Journal of Neuroscience, 28, 7209-7218.

Jenkinson, N., \& Brown, P. (2011). New insights into the relationship between dopamine, beta oscillations and motor function. Trends in Neurosciences, 34, 611-618.

Kühn, A. A., Williams, D., Kupsch, A., Limousin, P., Hariz, M., Schneider, G.-H., et al. (2004). Event-related beta desynchronization in human subthalamic nucleus correlates with motor performance. Brain, 127, 735-746.

Lachaux, J. P., Rodriguez, E., Martinerie, J., \& Varela, F. J. (1999). Measuring phase synchrony in brain signals. Human Brain Mapping, 8, 194-208.

Laming, D. R. J. (1979). Autocorrelation of choice-reaction times. Acta Psychologica, 43, 381-412.

Luce, R. D. (1986). Response times: Their role in inferring elementary mental organization. New York: Oxford University Press.

Mansfield, E. L., Karayanidis, F., Jamadar, S., Heathcote, A., \& Forstmann, B. U. (2011). Adjustments of response threshold during task switching: A model-based functional magnetic resonance imaging study. Journal of Neuroscience, 31, 14688-14692.

Marceglia, S., Fumagalli, M., \& Priori, A. (2011). What neurophysiological recordings tell us about cognitive and behavioral functions of the human subthalamic nucleus. Expert Review of Neurotherapeutics, 11, 139-149.

Miller, E. K., \& Cohen, J. D. (2001). An integrative theory of prefrontal cortex function. Annual Review of Neuroscience, 24, 167-202.

Mink, J. W. (1996). The basal ganglia: Focused selection and inhibition of competing motor programs. Progress in Neurobiology, 50, 381-425.

Nambu, A., Tokuno, H., \& Hamada, I. (2000). Excitatory cortical inputs to pallidal neurons via the subthalamic nucleus in the monkey. Journal of Neurophysiology, 84, 289-300.

Nambu, A., Tokuno, H., \& Takada, M. (2002). Functional significance of the cortico-subthalamo-pallidal "hyperdirect" pathway. Neuroscience Research, 43, 111-117. 
Rabbit, P. M. A. (1966). Errors and error correction in choiceresponse tasks. Journal of Experimental Psychology, 71, 264-272.

Ratcliff, R., \& Frank, M. J. (2012). Reinforcement-based decision making in corticostriatal circuits: Mutual constraints by neurocomputational and diffusion models. Neural Computation, 24, 1186-1229.

Ratcliff, R., \& McKoon, G. (2008). The diffusion decision model: Theory and data for two-choice decision tasks. Neural Computation, 20, 873-922.

Ridderinkhof, K. R., Ullsperger, M., Crone, E. A., \& Nieuwenhuis, S. (2004). The role of the medial frontal cortex in cognitive control. Science, 306, 443-447.

Rushworth, M. F. S., Noonan, M. P., Boorman, E. D., Walton, M. E., \& Behrens, T. E. (2011). Frontal cortex and rewardguided learning and decision-making. Neuron, 70, 1054-1069.

Simon, J. R., \& Rudell, A. P. (1967). Auditory S-R compatibility: The effect of an irrelevant cue on information processing. Journal of Applied Psychology, 51, 300-304.

Weinberger, M., Mahant, N., Hutchison, W. D., Lozano, A. M. Moro, E., Hodaie, M., et al. (2006). Beta oscillatory activity in the subthalamic nucleus and its relation to dopaminergic response in Parkinson's disease. Journal of Neurophysiology, 96, 3248-3256.

Wiecki, T. V., \& Frank, M. J. (2013). A computational model of inhibitory control in frontal cortex and basal ganglia. Psychological Review, 120, 329-355.

Wylie, S. A., Ridderinkhof, K. R., Elias, W. J., Frysinger, R. C., Bashore, T. R., Downs, K. E., et al. (2010). Subthalamic nucleus stimulation influences expression and suppression of impulsive behaviour in Parkinson's disease. Brain, 133, 3611-3624

Zaghloul, K. A., Weidemann, C. T., Lega, B. C., Jaggi, J. L., Baltuch, G. H., \& Kahana, M. J. (2012). Neuronal activity in the human subthalamic nucleus encodes decision conflict during action selection. Journal of Neuroscience, 32, $2453-2460$

Zavala, B., Brittain, J.-S., Jenkinson, N., Ashkan, K., Foltynie, T., Limousin, P., et al. (2013). Subthalamic nucleus local field potential activity during the Eriksen flanker task reveals a novel role for theta phase during conflict monitoring. Journal of Neuroscience, 33, 14758-14766. 